\title{
Effect of electroconvulsive stimulation on messenger RNA expression in the prefrontal cortex in a rat pain model
}

\author{
YUSUKE KIMURA $^{1}$, MASASHI ISHIKAWA ${ }^{1}$, YOKO HORI $^{1}$, TADASHI OKABE ${ }^{2}$ and ATSUHIRO SAKAMOTO ${ }^{1}$ \\ ${ }^{1}$ Department of Anesthesiology and Pain Medicine, Graduate School of Medicine, Nippon Medical School, \\ Tokyo 113-8603; ${ }^{2}$ Department of Anesthesiology, Hitachi, Ltd., Hitachinaka General Hospital, Ibaraki 312-0057, Japan
}

Received July 13, 2015; Accepted August 4, 2015

DOI: $10.3892 /$ br.2015.525

\begin{abstract}
Previous reports have shown that electroconvulsive therapy (ECT) is efficacious in the treatment of neuropathic pain; however, its mechanism of action remains unclear. The present study aimed to understand these mechanisms by investigating the alterations in the expression of neuropeptide $\mathrm{Y}$ $(N P Y)$ and interleukin- $1 \beta(I L-1 \beta)$ in the prefrontal cortex. A rat model of neuropathic pain produced by chronic constrictive injury of the sciatic nerve was used, and mechanical and thermal hyperalgesia were evaluated starting 2 days after the injury. Using a pulse generator, ECT was administered to the rodents for 6 days from days 7-12 after the injury. Thermal and mechanical stimulation were administered to assess pain thresholds. Quantitative polymerase chain reaction, used to measure gene expression levels in the prefrontal cortex, showed that $N P Y$ and $I L-1 \beta$ gene expression levels in the prefrontal cortex increased following the injury. The present results indicate that these gene expression level variations may be associated with the mechanisms underlying the effect of ECT in treating neuropathic pain.
\end{abstract}

\section{Introduction}

Electroconvulsive therapy (ECT) has been widely used as an effective treatment for schizophrenia and refractory depression; however, the underlying mechanism remains unclear. Numerous studies in the field of neuropsychiatry have indicated that the expression levels of genes encoding various neurotransmitters are altered following ECT, suggesting that these changes may be a possible mechanism of action of ECT (1-4). Certain studies have reported the efficacy of ECT in treating neuropathic pain (5-9). However, ECT is not widely

Correspondence to: Dr Yusuke Kimura, Department of Anesthesiology and Pain Medicine, Graduate School of Medicine, Nippon Medical School, 1-1-5 Sendagi, Tokyo 113-8603, Japan E-mail: yusuke-k@nms.ac.jp

Key words: electroconvulsive stimulation, prefrontal cortex, neuropeptide $\mathrm{Y}$, interleukin-1 $\beta$, messenger RNA used for treating neuropathic pain as the underlying mechanism of action has not been sufficiently elucidated.

Chronic pain causes changes in the expression of messenger RNAs (mRNAs) or proteins that regulate cytokines, neurotransmitters and receptors in the brain and dorsal root ganglia $(10,11)$. The differential expression of multiple pain-associated genes, such as interleukin- $1 \beta(I L-1 \beta)$, has an important role in the development and maintenance of chronic pain (12-15). Electroconvulsive stimulation (ECS) improves neuropathic pain symptoms in rats (16). In our previous report, altered expression of neuropeptide $\mathrm{Y}(N P Y)$ in the brain was identified as a possible mechanism of action of ECT for treating neuropathic pain (16).

The prefrontal cortex is known to participate in executive function, emotions and pain. Certain studies have shown prefrontal cortex dysfunction in chronic pain patients $(17,18)$, suggesting it has an important role in pain. However, there are only a few studies examining the participation of the prefrontal cortex in neuropathic pain and response to ECT. We hypothesized that changes in $N P Y$ and $I L-1 \beta$ gene expression levels in the prefrontal cortex may have an important role in the mechanisms underlying neuropathic pain and the effect of ECS in its treatment.

\section{Materials and methods}

Experimental animals. All the experimental procedures were approved by the Institutional Committee on Laboratory Animals of Nippon Medical School, Tokyo, Japan (approval no. 27-042) and were performed under the guidelines of the International Association for the Study of Pain (19).

Male Sprague-Dawley rats [6-7 weeks of age, $250 \mathrm{~g}$ (Tokyo Laboratory Animals Science Co., Tokyo, Japan)] were housed in clear, plastic cages with sawdust bedding at standard room temperature under a 12-h light/dark cycle. All the rats received food and water ad libitum. The rats were divided into three experimental groups: i) Rats subjected to the chronic constrictive injury (CCI) protocol and administered ECS (ECS group, $\mathrm{n}=7$ ); ii) those subjected to the CCI protocol with no ECS (CCI group, $n=7$ ); and iii) those subjected to a sham-CCI surgery (control group, $n=7$ ) (16).

Production of a neuropathic pain model. Experimental neuropathy was produced according to previously described 
methods (20). All the surgical procedures were performed on rats that were anesthetized with sodium pentobarbital [50 mg/kg intraperitoneally (i.p.)]. CCI was induced on the left (ipsilateral) side by exposing the common sciatic nerve in the left mid-thigh and loosely ligating the nerve using 4-0 silk thread in 4 regions spaced at $\sim 1-\mathrm{mm}$ intervals. As a control, the right (contralateral) sciatic nerve was similarly exposed, but not ligated. Rats in the control group were subjected to exposure of both sciatic nerves without nerve ligation.

ECS. ECS was administered transauricularly using metal forceps, as previously described $(1,2,21,22)$. ECS was administered to the ECS group once daily for 6 days, starting on day 7 postoperation and continuing to day 12 , using a pulse generator [frequency, 100 pulses; pulse width, $0.5 \mathrm{msec}$; shock duration, $0.8 \mathrm{sec}$; current, $50 \mathrm{~mA}$ (57800 ECT Unit; Ugo Basile, Comero, Italy)]. The shock elicited a full tonic-clonic seizure lasting $\sim 30 \mathrm{sec}$ in all rats. Rats in the CCI and control groups were exposed to the forceps once daily from days 7 to 12 postoperation; these animals, however, did not receive a current (16).

Behavioral tests. Two behavioral tests (thermal and mechanical stimulation) were performed 8 times to evaluate pain thresholds, as previously described $(22,23)$. They were performed the day before surgery (day 0) and on days 2, 4, 6, 8, 10, 12 and 14 after the surgery. During the period of ECS, the behavioral tests were performed subsequent to ECS (days 8 , 10 and 12) and $48 \mathrm{~h}$ after the last ECS (day 14). The plantar test (Ugo Basile) was used to analyze thermal allodynia. Rats were placed on a glass plate with radiant heat (a 50-W halogen reflector bulb) positioned underneath. Following an acclimation period, radiation heat was independently applied to either the ipsilateral or contralateral hind paw pad. The latency of paw withdrawal from thermal stimulus was measured 3 times at 5-min intervals, and the average value was used as the latency response. Mechanical hyperalgesia was measured using an electronic von Frey instrument (IITC Life Science, Woodland Hills, CA, USA). The instrument consisted of a disposable tip, a hand-held probe, a display unit and a connector cable. A polypropylene pipette tip was mounted on the probe and used for mechanical stimulation. Each rat was set on a metallic mesh floor and covered with a plastic box. Subsequently, the tip was applied from under the mesh floor to the plantar surface of either the ipsilateral or contralateral hind paw. When the rat felt pain and raised its leg, the mechanical stimuli was immediately stopped and the force shown on the digital display was recorded. Each paw was stimulated with the tip 3 times at 10 -sec intervals in individual trials, and the average value was used as the latency response (16).

Reverse transcription-quantitative polymerase chain reaction $(R T-q P C R)$. On day 14 postoperation, rats were anesthetized with pentobarbital at a dose of $300 \mathrm{mg} / 100 \mathrm{~g}$ i.p. before being sacrificed. The brains were immediately removed, and the prefrontal cortex, containing a coronal slice of the most anterior $3 \mathrm{~mm}$ of the brain (without the olfactory bulbs) was dissected as previously described (24). The prefrontal cortex was separated into right and left hemispheric regions. Each sample was placed in RNAlater ${ }^{\circledR}$ solution (Applied Biosystems,
Foster City, CA, USA) and stored at $-80^{\circ} \mathrm{C}$ until use. Prior to use, the samples were defrosted and the RNAlater ${ }^{\circledR}$ solution was immediately separated from the samples. Total RNA was extracted from the samples using an mirVana ${ }^{\mathrm{TM}}$ miRNA isolation $\mathrm{kit}^{\circledR}$ (Applied Biosystems), according to the manufacturer's instructions. RNA quality and quantity were evaluated by absorbance using a NanoDrop ND-1000 (Thermo Fisher Scientific, Waltham, MA, USA). Samples with an A260/280 ratio between 1.8 and 2.0 were of sufficient quality for use in subsequent analysis. Total RNA $(10 \mu \mathrm{g})$ was used as a template for RT reactions with $10 \mu \mathrm{l}$ of $10 \mathrm{X}$ RT buffer, $4 \mu \mathrm{l}$ of 25X deoxyribonucleotide triphosphate mix, $10 \mu \mathrm{l}$ of 10X RT random primers, $5 \mu \mathrm{l}$ of MultiScribe ${ }^{\mathrm{TM}} \mathrm{RT}, 5 \mu \mathrm{l}$ of RNase inhibitor and nuclease-free $\mathrm{H}_{2} \mathrm{O}$ (Applied Biosystems). The RT reaction was carried out using a PCR Express (Thermo Fisher Scientific, Yokohama, Japan) at $42^{\circ} \mathrm{C}$ for $60 \mathrm{~min}, 99^{\circ} \mathrm{C}$ for $10 \mathrm{~min}$ and $4^{\circ} \mathrm{C}$ for $5 \mathrm{~min}$.

The amplification of $N P Y$ and $I L-1 \beta$ was performed in a fast 96-well reaction plate (Applied Biosystems, Foster City, CA, USA), as previously described (25). Reactions were carried out in a $20-\mu 1$ volume containing $10 \mu \mathrm{l}$ of TaqMan Universal PCR master mix, $1 \mu$ l of TaqMan gene expression assays (Applied Biosystems), $8 \mu \mathrm{l}$ of RNase-free water (Wako, Tokyo, Japan) and $20 \mathrm{ng}$ of cDNA. The expression of glyceraldehyde-3-phosphate dehydrogenase (GAPDH) was used as an internal control to assess DNA integrity.

RT-qPCR consisted of an initial denaturation at $95^{\circ} \mathrm{C}$ for $3 \mathrm{sec}$, incubation at $60^{\circ} \mathrm{C}$ for $30 \mathrm{sec}$ and subsequent measurement of the fluorescence signal following each cycle. The TaqMan probe labeled with 6-fluorescein amidite was cleaved during amplification and generated a fluorescent signal. The assay used an instrument capable of measuring fluorescence in real-time (ABI PRISM 7500 Fast Sequence Detector; Applied Biosystems). RT-qPCR data are presented as threshold cycle $(\mathrm{Ct})$ values, where $\mathrm{Ct}$ indicates a unit-less value defined as the fractional cycle number at which the sample fluorescence signal passes a fixed threshold above baseline. Triplicate samples with significantly different values due to inaccurate operation were omitted. Relative amounts of all the mRNAs were calculated using the comparative $\mathrm{Ct}$ method (Applied Biosystems). $\Delta \mathrm{Ct}$ is the difference in the $\mathrm{Ct}$ values derived from the experimental samples and the GAPDH control, and $\Delta \Delta \mathrm{Ct}$ represents the difference between paired samples, as calculated by the following formula: $\Delta \Delta \mathrm{Ct}=(\Delta \mathrm{Ct}$ of targets of $\mathrm{CCI}+\mathrm{ECS}$ group) $-(\Delta \mathrm{Ct}$ of control of $\mathrm{CCI}+$ sham-ECS group) The expression ratio indicates the relative quantity of the target gene $\left(\mathrm{X}_{\text {target }}\right)$ to the control gene $\left(\mathrm{X}_{\text {control }}\right)$. The expression ratio was computed using the formula: $X_{\text {target }} / X_{\text {control }}=2^{-\Delta \Delta C t}$.

Statistical analysis. All the numerical data are expressed as mean \pm standard errors of the mean. A paired t-test was used to compare the latencies or threshold values in behavioural tests between the ipsilateral and contralateral sides (ECS group, $n=7$; CCI group; $n=7$ ). Dunnett's test for multiple comparisons was used to compare latencies, threshold values or different scores obtained in behavioural tests performed prior to the procedures (day $0, n=7$ ) with those obtained in tests performed following the procedures (days 2, 4, 6, 8, 10, 12 and 14; $n=7$ ). Analysis of variance followed by Tukey's test was performed using GraphPad Prism (GraphPad Software, Inc., La Jolla, CA, USA). 

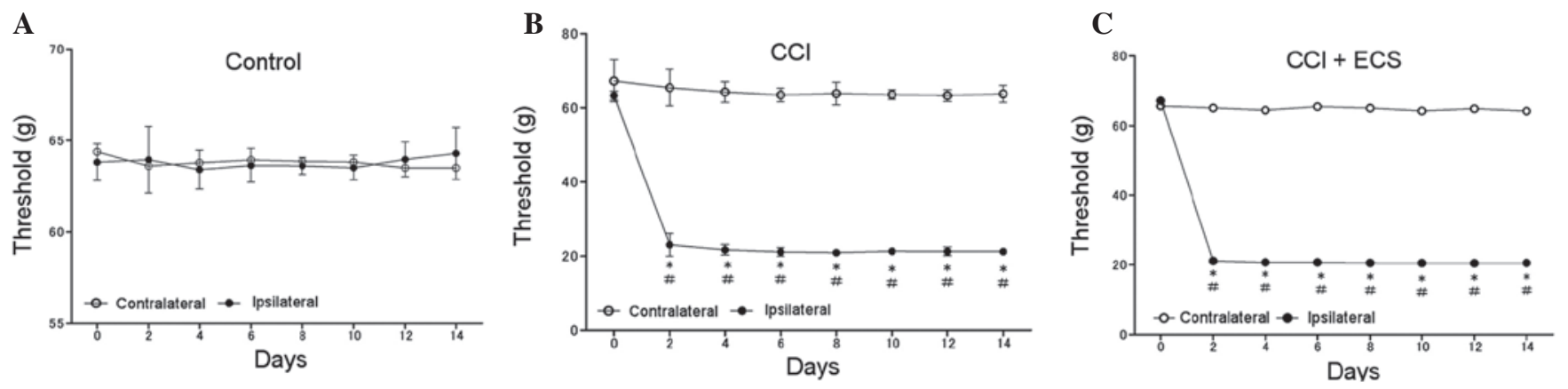

Figure 1. Effect of chronic constrictive injury (CCI) and electroconvulsive shock (ECS) on mechanical hyperalgesia in rats. Thresholds for paw withdrawal were recorded on the ipsilateral and contralateral sides in response to mechanical stimuli applied to the corresponding hind paw pad. (A) The ipsilateral and contralateral sides did not exhibit mechanical hyperalgesia in the control group. (B) The effects of the CCI procedure on mechanical hyperalgesia ( $\mathrm{n}=7$ ). (C) Mechanical hyperalgesia in $\mathrm{CCI}$ model rats administered $\mathrm{ECS}(\mathrm{n}=7)$. ${ }^{\mathrm{P}} \mathrm{P}<0.05$, compared with values from the contralateral side on the same days, using a paired $\mathrm{t}$-test; $\mathrm{P}<0.05$, compared with values on the preoperative day (day 0), using Dunnett's multiple comparisons. Values are presented as mean \pm standard errors of the mean.
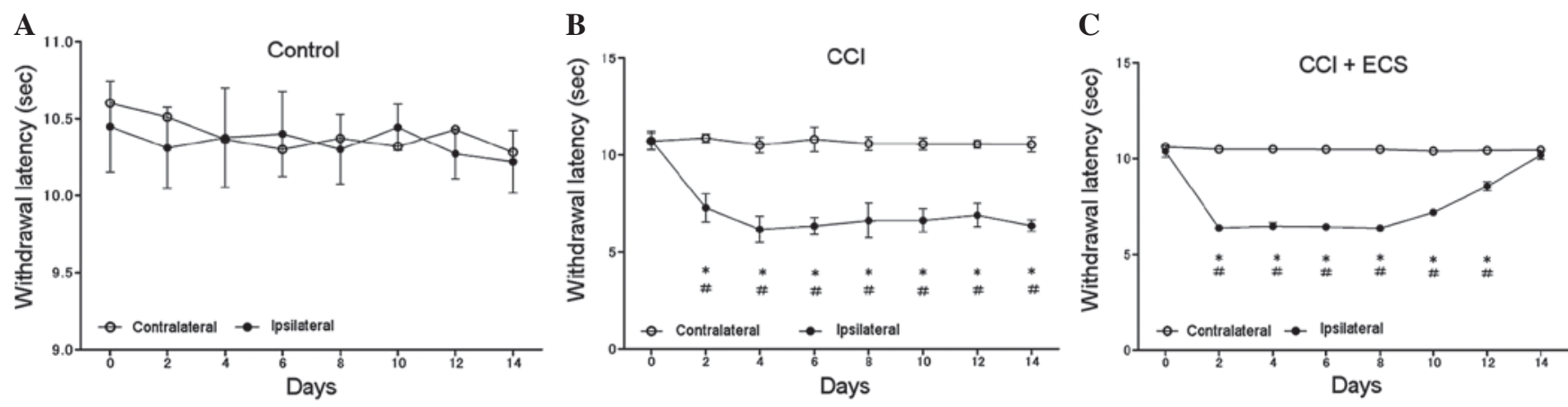

Figure 2. Effect of chronic constrictive injury (CCI) and electroconvulsive shock (ECS) on thermal allodynia in rats. Thresholds for paw withdrawal were recorded on the ipsilateral and contralateral sides in response to thermal stimuli applied to the corresponding hind paw pad. (A) The ipsilateral and contralateral sides did not exhibit thermal allodynia in the control group. (B) The effects of the CCI procedure on thermal allodynia ( $\mathrm{n}=7$ ). (C) Thermal allodynia in CCI model rats administered ECS $(n=7)$. The degree of reduction in thermal allodynia by ECS is statistically significant $($ day 14$)$. ${ }^{*}<0.05$, compared with values on the contralateral side on the same days using a paired t-test; ${ }^{\#} \mathrm{P}<0.05$, compared with the values on the preoperative day (day 0 ) using Dunnett's multiple comparisons. Values are presented as mean \pm standard errors of the mean.

\section{Results}

Behavioral tests. There was no difference in the latency of paw withdrawal in the plantar test and threshold values in the electronic von Frey test between the ipsilateral and contralateral sides in the control group. The CCI group showed a significant decrease in the latency of paw withdrawal in the plantar test and threshold values in the electronic von Frey test for the ipsilateral side on postoperative days $(2,4,6,8,10$, 12 and 14) compared with the corresponding values on day 0 $(\mathrm{P}<0.01)$. However, significant decreases were not observed on the contralateral side. Paw withdrawal thresholds in response to mechanical stimulation were not significantly altered following ECS. Compared with the values on day 0, in the ECS group, latencies of paw withdrawal from thermal stimulation on the ipsilateral side were decreased on days 2, 4, 6, 8 and $10(\mathrm{P}<0.01)$. After ECS, the latencies of paw withdrawal from thermal stimulation on the ipsilateral side were increased on days 12 and 14 (Figs. 1 and 2).

$R T$-qPCR of mRNAs. In the right prefrontal cortex, expression of $N P Y$ was 1.7 times greater in the CCI group compared with the ECS and control groups $(\mathrm{P}<0.01)$; expression levels in the ECS and control groups were similar. No significant differences among the 3 groups were observed in the left prefrontal cortex. In the right prefrontal cortex, the expression of $I L-1 \beta$ was 1.4 times greater in the CCI group compared with the ECS and control groups $(\mathrm{P}<0.01)$. In addition, expression of $I L-1 \beta$ in the ECS group was lower compared with the control group. In the left prefrontal cortex, there were no significant differences observed in $I L-1 \beta$ gene expression between the $\mathrm{CCI}$ and control groups. However, $I L-1 \beta$ gene expression was lower in the ECS group compared with the control group $[\mathrm{P}<0.01$ (Fig. 3)].

\section{Discussion}

Functional brain imaging has revealed that the mechanism of pain is not simply an alteration of sensory neural transmission from the periphery to the cerebrum, such as in the spinothalamic tract. With respect to chronic pain, it has been suggested that shifting the focus from 'an infringed sensory system' to 'an emotion system' is essential for advancing mechanistic understanding (26). In addition, it is thought that pain arising from damage and inflammation is associated with mental and physical stress (27). Mental stress has been shown to activate the cerebral cortex (such as prefrontal cortex) and limbic system prior to spreading to the central 

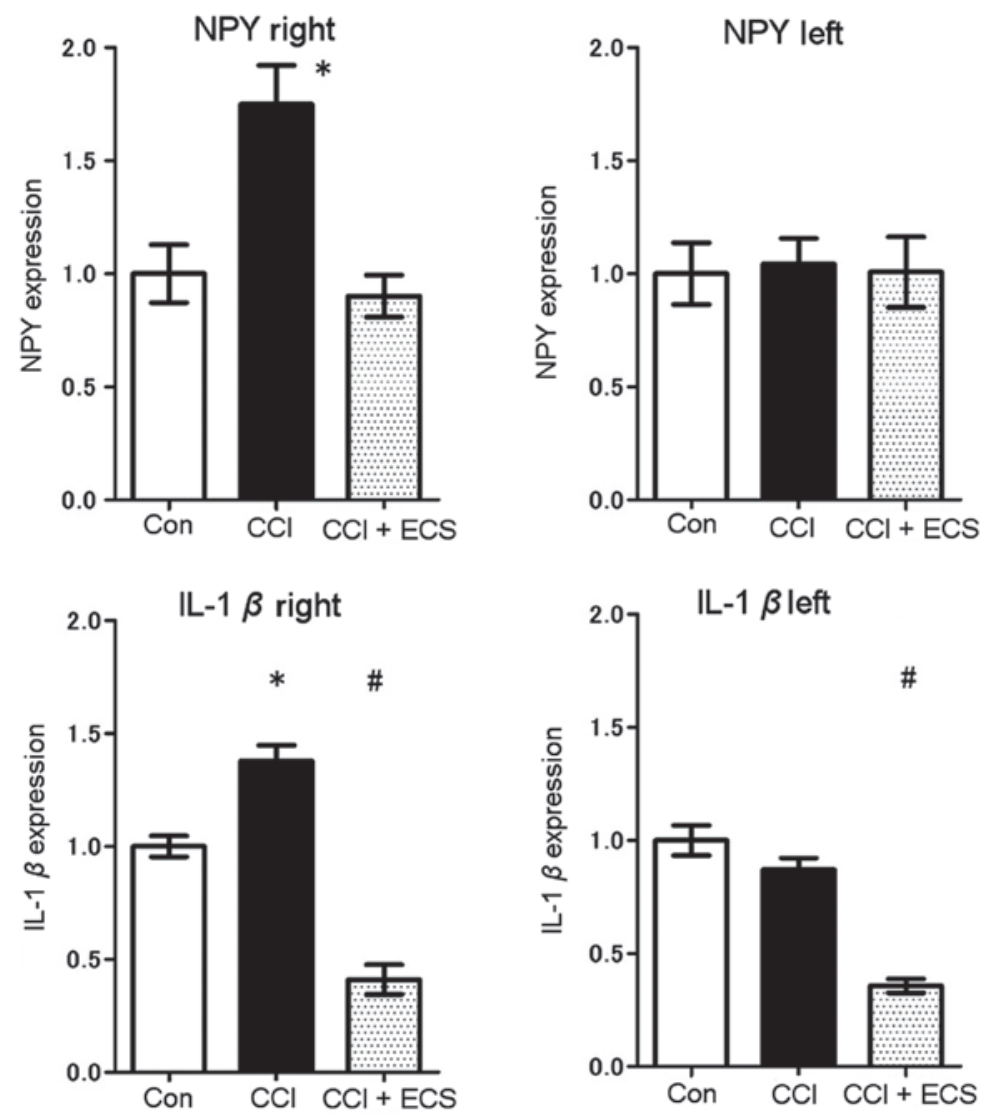

Figure 3. Effect of chronic constrictive injury (CCI) and electroconvulsive shock (ECS) on neuropeptide Y (NPY) expression in rat prefrontal cortex. In the right prefrontal cortex, the expression of NPY in the CCI group $(n=7)$ was significantly greater compared to in the ECS $(n=7)$ and control groups $(n=7)$. Additionally, the expression of NPY in the ECS and control groups was similar. Conversely, there was no significant difference among the three groups in the left prefrontal cortex. The effect of CCI and ECS on interleukin-1 $\beta$ (IL-1ß) expression in rat prefrontal cortex. In the right prefrontal cortex, the expression of IL-1 $\beta$ in the CCI group ( $n=7)$ was significantly greater than that in the ECS $(n=7)$ and control groups $(n=7)$. Additionally, the expression of IL-1 $\beta$ was lower in the ECS group compared to in the control group. Conversely, the expression of IL-1 $\beta$ in the left prefrontal cortex was similar in the CCI and control groups, whereas it was lower in the ECS group compared to in the control group. " $\mathrm{P}<0.01$ compared with the expression levels in the control group using Dunnett's multiple comparisons. ${ }^{~} \mathrm{P}<0.01$ compared with the expression levels in the control group using Dunnett's multiple comparisons. Values are presented as means \pm standard errors of the mean.

amygdaloid nucleus or bed nucleus of stria terminalis (BST), which results in the activation of the hypothalamo-pituitary-adrenocortical axis (28). Although it is known that $N P Y$ is associated with appetite, blood pressure and memory, it has also been recently suggested that $N P Y$ could be involved in pain (29). Furthermore, BST contains neurons that are associated with the unpleasantness of pain, and it was also reported that $N P Y$ controls the function of these neurons (30). On the basis of the above data, the present study was interpreted to find that elevated $N P Y$ expression in the prefrontal cortex of the CCI group normalized to control levels in the ECS group according to the following hypothesis: Activity of neurons associated with pain in BST was promoted by the stress associated with CCI, and NPY gene expression increased in the prefrontal cortex as a compensatory response. As pain was decreased by ECS, it is thought that NPY expression correspondingly returned to control values in the prefrontal cortex. Briefly, the alterations in NPY expression between the prefrontal cortex and BST were thought to occur to control the CCI-induced mental stress. NPY expression was altered in the right prefrontal cortex (as the left sciatic nerve was ligated) as it utilizes the spinothalamic tract, which innervates the contralateral side of the spinal cord.
The effects of $I L-1 \beta$ are varied and are recognized as important for controlling immunity and inflammation. Apkarian et al (24) showed that $I L-1 \beta$ mRNA expression in the prefrontal cortex increased in the spinal nerve injury model rat. Although a similar alteration in $I L-1 \beta$ expression following CCI was not reported, the study also failed to obtain effective allodynia in the CCI model rats. In the present study, effective allodynia was achieved in the CCI model rats and provided results that were equivalent to their study in CCI model rats. The neuropathic pain symptoms were improved by performing ECS as expression of $I L-1 \beta$ decreased in the ECS group. Furthermore, the laterality of $I L-1 \beta$ expression was consistent with that of $N P Y$ expression. Although it was expected that the alterations in expression would be normalized to control levels following ECS, as with $N P Y$, the expression of $I L-1 \beta$ fell to levels below that of the control group. Notably, this phenomenon was observed in the left and right prefrontal cortex.

The results of the present study indicate that ECS is effective for curing thermal allodynia, but not for treating mechanical hyperalgesia. This result is similar to those of previous studies $(16,22)$. Up to postoperative day 10 , the development of thermal allodynia and mechanical hyperalgesia was shown on the affected side in the CCI and ECS groups, and 
a significant difference was corroborated between the preoperative and postoperative data. As there was not a significant difference in the scores of the thermal and mechanical stimulant tests between the 2 groups, it can be suggested that there was no difference in the levels of CCI between the two groups.

In conclusion, the present study showed that the symptoms of neuropathic pain improved, and the level of expression of the $N P Y$ and $I L-1 \beta$ genes in the prefrontal cortex decreased in CCI model rats following ECS. The $N P Y$ and $I L-1 \beta$ gene expression levels in the prefrontal cortex were increased in CCI model rats. The possibility exists that changes in the levels of expression of $N P Y$ and $I L-1 \beta$ in the prefrontal cortex are associated with the mechanism of action of ECT in treating neuropathic pain.

\section{References}

1. Mikkelsen JD and Woldbye DP: Accumulated increase in neuropeptide $\mathrm{Y}$ and somatostatin gene expression of the rat in response to repeated electroconvulsive stimulation. J Psychiatr Res 40: 153-159, 2006.

2. Ploski JE, Newton SS and Duman RS: Electroconvulsive seizure-induced gene expression profile of the hippocampus dentate gyrus granule cell layer. J Neurochem 99: 1122-1132, 2006

3. Altar CA, Laeng P, Jurata LW, Brockman JA, Lemire A, Bullard J, Bukhman YV, Young TA, Charles V and Palfreyman MG: Electroconvulsive seizures regulate gene expression of distinct neurotrophic signaling pathways. J Neurosci 24: 2667-2677, 2004

4. Shen H, Numachi Y, Yoshida S, Fujiyama K, Toda S, Awata S, Matsuoka H and Sato M: Electroconvulsive shock increases serotonin transporter in the rat frontal cortex. Neurosci Lett 341: 170-172, 2003

5. Mandel MR: Electroconvulsive therapy for chronic pain associated with depression. Am J Psychiatry 132: 632-636, 1975.

6. Bloomstein JR, Rummans TA, Maruta T, Lin SC and Pileggi TS: The use of electroconvulsive therapy in pain patients. Psychosomatics 37: 374-379, 1996.

7. King JH and Nuss S: Reflex sympathetic dystrophy treated by electroconvulsive therapy: Intractable pain, depression, and bilateral electrode ECT. Pain 55: 393-396, 1993.

8. Rasmussen KG and Rummans TA: Electroconvulsive therapy for phantom limb pain. Pain 85: 297-299, 2000.

9. Rasmussen KG and Rummans TA: Electroconvulsive therapy in the management of chronic pain. Curr Pain Headache Rep 6: $17-22,2002$.

10. Lee HL, Lee KM, Son SJ, Hwang SH and Cho HJ: Temporal expression of cytokines and their receptors mRNAs in a neuropathic pain model. Neuroreport 15: 2807-2811, 2004.

11. Urban MO and Gebhart GF: Supraspinal contributions to hyperalgesia. Proc Natl Acad Sci USA 96: 7687-7692, 1999.

12. Kuss AW and Chen W: MicroRNAs in brain function and disease. Curr Neurol Neurosci Rep 8: 190-197, 2008.

13. Farh KK, Grimson A, Jan C, Lewis BP, Johnston WK, Lim LP, Burge CB and Bartel DP: The widespread impact of mammalian MicroRNAs on mRNA repression and evolution. Science 310: 1817-1821, 2005.

14. Lim LP, Lau NC, Garrett-Engele P, Grimson A, Schelter JM, Castle J, Bartel DP, Linsley PS and Johnson JM: Microarray analysis shows that some microRNAs downregulate large numbers of target mRNAs. Nature 433: 769-773, 2005.
15. Alexander GM, van Rijn MA, van Hilten JJ, Perreault MJ and Schwartzman RJ: Changes in cerebrospinal fluid levels of pro-inflammatory cytokines in CRPS. Pain 116: 213-219, 2005.

16. Okabe T, Sato C, Matsumoto K, Ozawa H and Sakamoto A: Electroconvulsive stimulation (ECS) increases the expression of neuropeptide Y (NPY) in rat brains in a model of neuropathic pain: A quantitative real-time polymerase chain reaction (RT-PCR) study. Pain Med 10: 1460-1467, 2009.

17. Apkarian AV, Sosa Y, Sonty S, Levy RM, Harden RN, Parrish TB and Gitelman DR: Chronic back pain is associated with decreased prefrontal and thalamic gray matter density. J Neurosci 24: 10410-10415, 2004.

18. Schmidt-Wilcke T, Leinisch E, Gänssbauer S, Draganski B, Bogdahn U, Altmeppen J and May A: Affective components and intensity of pain correlate with structural differences in gray matter in chronic back pain patients. Pain 125: 89-97, 2006.

19. Covino BG, Dubner R, Gybels J, Kosterlitz HW, Liebeskind JC, Sternbach RA, Vyklicky L, Yamamura $\mathrm{H}$ and Zimmermann M; The Committee for Research and Ethical Issues of the International Association for the Study of Pain: Ethical standards for investigations of experimental pain in animals. Pain 9: 141-143, 1980.

20. Bennett GJ and Xie YK: A peripheral mononeuropathy in rat that produces disorders of pain sensation like those seen in man. Pain 33: 87-107, 1988.

21. Woldbye DP, Greisen MH, Bolwig TG, Larsen PJ and Mikkelsen JD: Prolonged induction of c-fos in neuropeptide Y-and somatostatin-immunoreactive neurons of the rat dentate gyrus after electroconvulsive stimulation. Brain Res 720: 111-119, 1996.

22. Shibata M, Wakisaka S, Inoue T, Shimizu T and Yoshiya I: The effect of electroconvulsive treatment on thermal hyperalgesia and mechanical allodynia in a rat model of peripheral neuropathy. Anesth Analg 86: 584-587, 1998.

23. Sato C, Sakai A, Ikeda Y, Suzuki H and Sakamoto A: The prolonged analgesic effect of epidural ropivacaine in a rat model of neuropathic pain. Anesth Analg 106: 313-320, 2008.

24. Apkarian AV, Lavarello S, Randolf A, Berra HH, Chialvo DR, Besedovsky HO and del Rey A: Expression of IL-1beta in supraspinal brain regions in rats with neuropathic pain. Neurosci Lett 407: 176-181, 2006.

25. Takemori K, Kobayashi K and Sakamoto A: Expression of pulmonary vasoactive factors after sevoflurane anaesthesia in rats: A quantitative real-time polymerase chain reaction study. Br J Anaesth 100: 190-194, 2008.

26. Hashmi JA, Baliki MN, Huang L, Baria AT, Torbey S, Hermann KM, Schnitzer TJ and Apkarian AV: Shape shifting pain: Chronification of back pain shifts brain representation from nociceptive to emotional circuits. Brain 136: 2751-2768, 2013.

27. Senba E and Ueyama T: Stress-induced expression of immediate early genes in the brain and peripheral organs of the rat. Neurosci Res 29: 183-207, 1997.

28. Herman JP and Cullinan WE: Neurocircuitry of stress: Central control of the hypothalamo-pituitary-adrenocortical axis. Trends Neurosci 20: 78-84, 1997.

29. Hökfelt T, Brumovsky P, Shi T, Pedrazzini T and Villar M: NPY and pain as seen from the histochemical side. Peptides 28: 365-372, 2007.

30. Ide S, Hara T, Ohno A, Tamano R, Koseki K, Naka T, Maruyama C, Kaneda K, Yoshioka M and Minami M: Opposing roles of corticotropin-releasing factor and neuropeptide $\mathrm{Y}$ within the dorsolateral bed nucleus of the stria terminalis in the negative affective component of pain in rats. J Neurosci 33: 5881-5894, 2013. 